\title{
Les moins de 30 ans en Gironde : regards croisés entre insertion et RSA
}

The under-30s in Gironde: a cross-section between integration and RSA

Mehdi Le Petit-Guerin et Khalid Eljim

\section{(2) OpenEdition}

\section{Journals}

Édition électronique

URL : https://journals.openedition.org/popvuln/819

DOI : $10.4000 /$ popvuln. 819

ISSN : 2650-7684

\section{Éditeur}

LIR3S - Laboratoire Interdisciplinaire de Recherche "Sociétés Sensibilités Soin" (UMR 7366 CNRS-uB)

Édition imprimée

Date de publication : 1 juin 2018

Pagination : $95-113$

ISBN : 978-2-918173-23-6

ISSN : 2269-0182

Référence électronique

Mehdi Le Petit-Guerin et Khalid Eljim, «Les moins de 30 ans en Gironde : regards croisés entre

insertion et RSA », Populations vulnérables [En ligne], 4 | 2018, mis en ligne le 12 juillet 2019, consulté le 26 février 2022. URL : http://journals.openedition.org/popvuln/819; DOI : https://doi.org/10.4000/ popvuln.819

Ce document a été généré automatiquement le 26 février 2022.

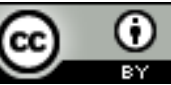

Les contenus de la revue Populations vulnérables sont mis à disposition selon les termes de la Licence Creative Commons Attribution 4.0 International 


\section{Les moins de 30 ans en Gironde : regards croisés entre insertion et RSA}

The under-30s in Gironde: a cross-section between integration and RSA

Mehdi Le Petit-Guerin et Khalid Eljim

\section{Introduction}

1 Entre études supérieures, exclusion, intérim, chômage et réorientation, l'insertion professionnelle des jeunes est un processus de moins en moins linéaire et prévisible. Face à ce défi sans cesse renouvelé, les acteurs du marché de l'emploi en France cherchent à enrayer les problématiques spécifiques à l'insertion socio-professionnelle des moins de 30 ans. Au premier rang de ces acteurs, l'État joue un rôle prépondérant en définissant, au sein de la jeunesse tout entière, quels sont les jeunes qui doivent pouvoir être soutenus par la solidarité collective.

Deux grandes périodes se succèdent dans l'histoire récente de la protection sociale française de la jeunesse. La première, dite du learnfare, mit l'accent sur l'amélioration du niveau de formation des jeunes, notamment en conditionnant l'octroi de l'aide à la poursuite d'études et à l'obtention de diplômes. Il s'agissait alors de politiques centrées autour de ce qui était considéré à l'époque comme le principal facteur de précarité chez les jeunes adultes: le décrochage scolaire. Progressivement mise à mal par un questionnement général sur le juste équilibre entre droits et obligations, l'assistance apportée jusque-là aux jeunes est remise en question à partir de la fin des années 1980 . Traditionnellement marquée par l'exclusion des jeunes du RMI en 1988 et la création du Fonds d'aide aux jeunes (FAJ) l'année suivante, l'activation des jeunes devient d'ordre professionnel (Lima, 2012). Depuis, les débats autour de la «désincitation au travail» ont entraîné la refonte d'une partie du système de protection sociale en conditionnant l'aide publique à l'emploi, et ce de manière encore plus stricte lorsqu'il s'agit de l'octroyer à un(e) jeune. Cette mutation de l'État social s'appuie sur une perception de 
la solidarité dite «active ", dont l'un des dispositifs les plus symboliques est le Revenu de solidarité active (RSA).

3 La structure relativement jeune de la population du département girondin, combinée aux outils de suivi des bénéficiaires du RSA mis en place très tôt sur le territoire, sont autant de raisons de d'investiguer le sujet à l'échelle de la Gironde. La première partie de cette recherche vise à caractériser les jeunes en situation d'exclusion dans le département. Les jeunes non insérés sont par définition des jeunes qui n'occupent pas d'emploi et ne sont pas inscrits dans un établissement pour suivre des études. Ils sont ainsi exclus du marché de l'emploi et du système scolaire. La seconde partie quant à elle opère un suivi de "jeunes » dont la situation précaire s'illustre par un ou plusieurs passage(s) dans le dispositif de RSA. Considérant que l'insertion des jeunes adultes relève d'un processus complexe et relativement long, cette étude mêle données de contextualisation et suivi de cohorte, afin de déterminer les tendances récentes de l'insertion des jeunes.

\section{Définition de la non-insertion des jeunes girondins}

4 Conséquence probable de la dernière crise, depuis 2009 les jeunes en France comme ailleurs sont de plus en plus touchés par les phénomènes d'exclusion (OCDE, 2016). Parmi les multiples réalités qu'englobe le terme d'exclusion, on désigne comme jeunes non insérés les personnes qui ne sont pas inscrites dans un établissement d'enseignement secondaire ou supérieur et qui n'ont pas d'emploi, fût-il occasionnel. La proportion de jeunes non insérés est le rapport de l'effectif des jeunes dans cette situation à l'ensemble de la population du groupe d'âge et constitue un premier indicateur central dans l'analyse de l'insertion des jeunes.

\section{Les jeunes de moins de 30 ans : de plus en plus d'exclus!}

Après être restée stable (16\%) entre 2006 et 2009, la proportion des jeunes girondins non insérés dépasse $18 \%$ en 2014, soit plus d'un jeune de 18 à 29 ans sur six. Cette moyenne cache néanmoins de fortes disparités par âge et par sexe. S'agissant de l'âge, ce phénomène est plus marqué chez les jeunes de plus de 25 ans. En 2014, dans ce groupe d'âge, plus d'un jeune sur cinq $(21,6 \%)$ est non inséré, soit près de six points de pourcentage de plus que chez les jeunes de 18 à 24 ans (16,1\%). Il apparait que la situation des jeunes girondins est légèrement favorable comparativement à la moyenne métropolitaine. Parmi les 18-24 ans, la proportion des non insérés à l'échelle du département est nettement inférieure à la moyenne métropolitaine (respectivement $16,1 \%$ et $18,8 \%$ ). Pour les jeunes de $25-29$ ans, même si la situation en Gironde est toujours favorable, l'écart avec la moyenne métropolitaine (respectivement $21,6 \%$ et $22,4 \%)$ est moindre. Cette situation relativement spécifique peut, entre autres, s'expliquer par une densité plus importante d'établissements d'enseignement supérieur dans le département. 
Figure 1. Évolution 2006-2014 de la proportion des jeunes de 18-29 ans non insérés, par groupe d'âges en Gironde

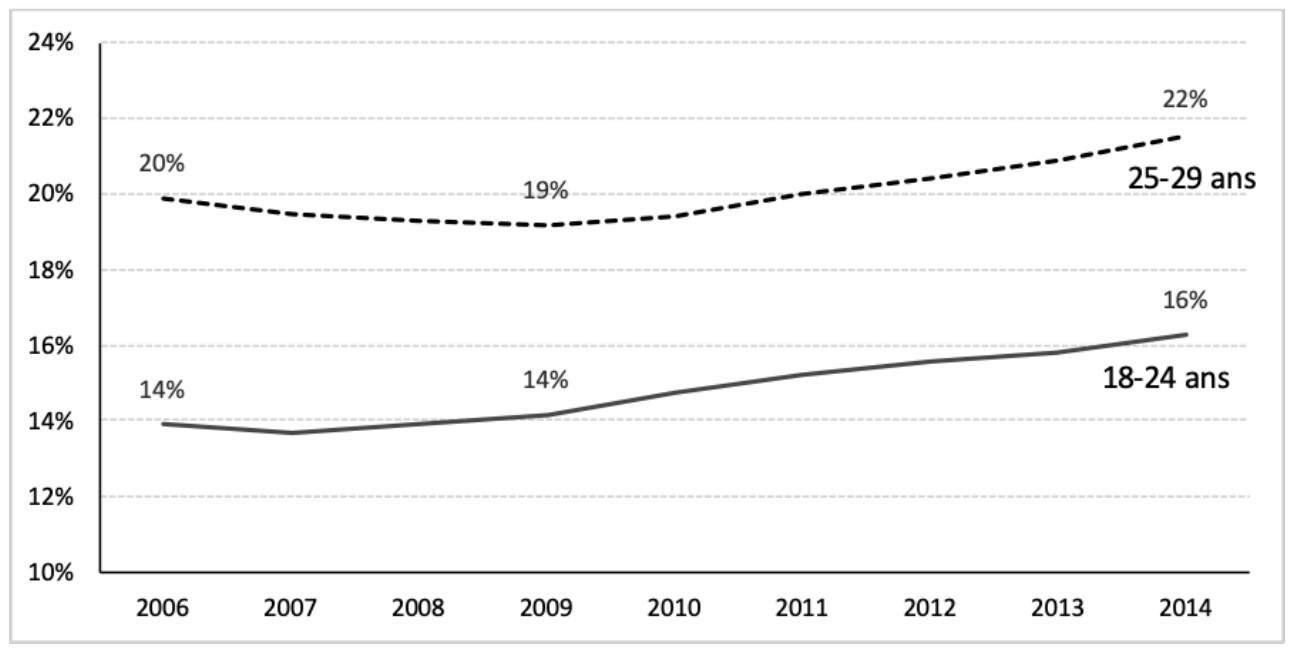

Traitement : auteurs

Source : INSEE-Enquêtes annuelles de recensement 2006 à 2014

6 Par ailleurs, les hommes et les femmes ne sont pas touchés dans les mêmes proportions. Considérant l'ensemble des jeunes de moins de 30 ans, les femmes apparaissent plus souvent non insérées que les hommes, les parts de personnes non insérées étant respectivement de $19 \%$ et $18 \%$. Toutefois, les tendances et les écarts varient nettement selon le groupe d'âges.

Parmi les jeunes de 18 à 24 ans, on observe depuis 2009, un renversement de situation : depuis lors, avec un écart qui ne cesse de se creuser, ce sont les jeunes hommes qui sont de moins en moins insérés. Alors qu'en 2008 la proportion des jeunes non insérés était de $14 \%$ aussi bien parmi les hommes que parmi les femmes de ce groupe d'âges, en 2014 , cette proportion atteint $17 \%$ chez les hommes et $15 \%$ chez les femmes.

Chez les jeunes de plus de 25 ans, la configuration de l'exclusion selon le sexe est restée pratiquement inchangée. Depuis 2006 ce sont les jeunes femmes qui sont et restent les plus touchées par ce problème. Toutefois, depuis 2009, du fait d'une détérioration plus accélérée de la situation des hommes, l'écart homme-femme s'est relativement résorbé.

$\mathrm{Au}$ final, les comparaisons par sexe et par âge montrent que chez les jeunes hommes, l'âge apparaît de moins en moins comme un facteur déterminant. Actuellement, le niveau d'exclusion chez les hommes de moins de 25 ans ne diffère pas sensiblement de celui observé chez les hommes de 25-29 ans (respectivement 17,2\% contre 19,1\%). A contrario, chez les femmes, ces écarts sont nettement plus importants (respectivement $15,4 \%$ chez les $18-24$ ans contre $23,9 \%$ dans le groupe des $25-29$ ans). 
Figure 2. Évolution 2006-2014 de la proportion des jeunes de 18-29 ans non insérés, par groupe d'âges et par sexe en Gironde

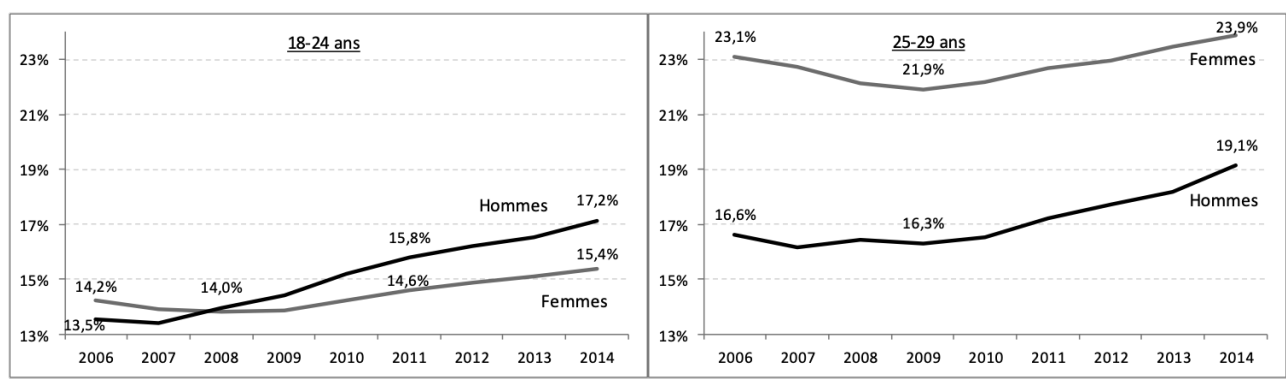

Traitement : auteurs

Source : INSEE-Enquêtes annuelles de recensement 2006 à 2014

\section{Les jeunes : de l'insertion par l'étude à l'exclusion par le chômage}

Les constats en termes de non-insertion conduisent à observer les tendances des jeunes femmes et hommes concernant la poursuite d'études et l'exposition au chômage.

11 En matière de poursuite d'études, les écarts entre les femmes et les hommes s'observent essentiellement parmi les jeunes de 18-24 ans. Dans ce groupe d'âges, alors que près d'une femme sur deux ( $48 \%$ ) est encore élève ou étudiante, les hommes ne sont que quatre sur dix $(41 \%)$ à poursuivre encore des études. Cet écart est quasiinexistant dans le groupe des 25-29 ans, où la proportion des jeunes qui poursuivent encore leurs études est de 5,1\% chez les femmes et 4,6\% chez les hommes. Les femmes semblent donc « protégées " plus longtemps que les hommes de la non-insertion par la poursuite d'études.

12 Le taux de chômage est nettement plus élevé chez les 18-24 ans, mais depuis 2006 la tendance est à la hausse parmi tous les jeunes, tous sexes et groupes d'âge confondus. Cependant les femmes sont surexposées au chômage par rapport à leurs homologues masculins et ce de manière plus forte entre 25 et 29 ans.

Figure 3. Évolution 2006-2014 du taux de chômage (au sens du recensement) des jeunes de 18-29 ans, par groupe d'âges et par sexe en Gironde

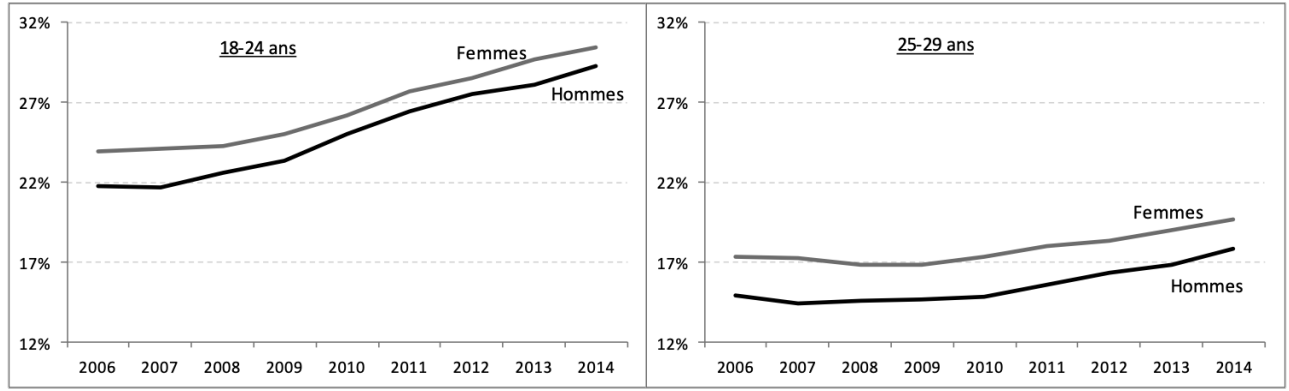

Traitement : auteurs

Source : INSEE-Enquêtes annuelles de recensement 2006 à 2014

13 Ces constats sont d'autant plus saisissants que les jeunes qui échappent à l'exclusion occupent des emplois de plus en plus précaires. En 2014, la précarité touche ainsi 33,4\% 
des jeunes de 18-29 ans occupant un emploi. Cette proportion était relativement moins élevée en 2008 (31,6\%). Par groupe d'âge, $48 \%$ des jeunes de moins de 24 ans occupent un emploi précaire. Cette proportion n'est « que » de $22 \%$ chez les jeunes de 25-29 ans. Par sexe la précarité touche d'avantage les jeunes femmes (35\%) que les jeunes hommes (32\%). À noter enfin que les jeunes occupant un emploi (y compris sous apprentissage ou en stage rémunéré) sont de plus en plus indépendants et de moins en moins salariés.

Les dynamiques de non-insertion chez les jeunes girondins apparaissent donc préoccupantes. Alors que les jeunes femmes de moins de 25 ans semblent mieux protégées de la non-insertion par la poursuite d'études, elles sont par la suite plus souvent en difficulté sur le marché du travail que les jeunes hommes. Les problématiques de l'emploi concernent néanmoins les jeunes de tous sexes et posent la question de l'insertion, et de la réinsertion, des jeunes adultes. En ce sens, les travaux suivants tentent d'identifier les problématiques d'insertion spécifiques aux jeunes adultes en s'appuyant sur un suivi de cohorte de bénéficiaires du RSA mené en Gironde.

\section{La précarité des jeunes au prisme du dispositif du RSA}

Entré en vigueur en juin 2009, le RSA prend la forme d'un revenu minimum versé pour : "assurer des moyens convenables d'existence", "lutter contre la pauvreté", " encourager l'exercice ou le retour à une activité professionnelle et aider à l'insertion sociale» (art. 1 de la loi n 2008-1249 instaurant le RSA). À la fois en soutenant les ménages sans ressources et en complétant les revenus des travailleurs pauvres, il vise donc un lien fort entre travail et insertion. Parallèlement au déploiement du dispositif sur le territoire, la Gironde a mis en place des outils d'analyse et de suivi des bénéficiaires du RSA permettant une évaluation efficace du dispositif. Le rapport 2016 de l'Observatoire girondin de la pauvreté et de la précarité (OGPP) relève qu'en Gironde la part de la population couverte par le RSA a augmenté de $36 \%$ de 2009 à 2015 . Cette augmentation significative suit de près la tendance pour la France métropolitaine, tout en restant légèrement inférieure dans le département (9,5\% en France métropolitaine contre $9 \%$ en Gironde en 2015).

La comparaison des structures par sexe et âge des bénéficiaires du RSA en Gironde d'une part et de l'ensemble de la population girondine âgée de 20 à 64 ans d'autre part, montre une nette surreprésentation des jeunes de 25-29 ans parmi les bénéficiaires (Figure 4). Ainsi, alors que les jeunes de $25-29$ ans représentent $11 \%$ de la population girondine (de 20 à 64 ans), ils constituent $21 \%$ des girondins bénéficiant du RSA. De plus, si de manière générale les femmes sont surreprésentées (56\%) parmi l'ensemble des bénéficiaires du RSA, elles le sont encore davantage $(60 \%)$ au sein des bénéficiaires âgés de 18 à 29 ans. Cette surreprésentation féminine est observée tout particulièrement parmi les bénéficiaires de moins de 25 ans dont $77 \%$ sont des femmes. 
Figure 4. Pyramides des âges des bénéficiaires du RSA en Gironde et de la population girondine (proportions pour 1000 personnes âgées de 20 à 64 ans) en 2015

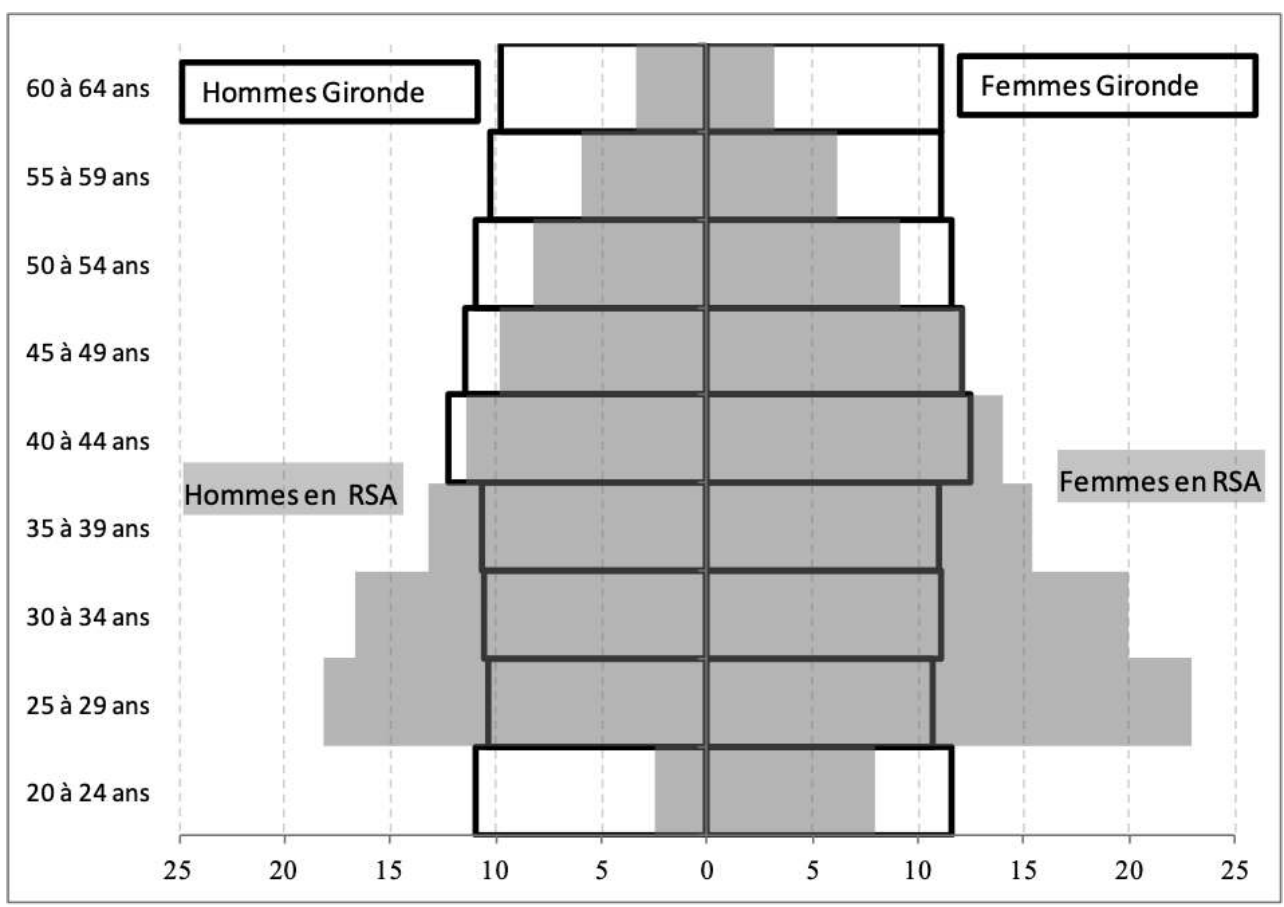

Traitement : auteurs

Source : Conseil départemental de la Gironde, extraction SID/INSEE-Enquêtes annuelles de recensement 2015

17 En Gironde, comme à l'échelle de la France métropolitaine, le nombre de jeunes de 18-29 ans bénéficiaires ${ }^{2}$ du RSA est en augmentation depuis sa mise en place. À la fin de l'année 2015, on compte près de 22000 bénéficiaires dans le département, soit $20 \%$ de plus qu'en $2011^{3}$. Cette augmentation est essentiellement due à l'augmentation du nombre de bénéficiaires âgés de 25-29 ans (Figure 5). Afin d'approfondir l'analyse des disparités de recours au RSA, nous avons calculé des "taux » de bénéficiaires par sexe et par âge (Figure 5). Au sein des 25-29 ans en Gironde, le recours au RSA est plus fréquent parmi les femmes que parmi les hommes, et relativement élevé pour les deux sexes, ce qui traduit les tendances de jeunes non-insérés évoquées précédemment. L'analyse des taux chez les moins de 25 ans appelle cependant quelques remarques. Certes ces jeunes recourent globalement moins au RSA car ils sont moins touchés par l'exclusion que les 25-29 ans. Cependant le recours est resté stable depuis 2011, signe que malgré l'augmentation de l'exclusion sur la même période, cela n'a pas engendré une augmentation du recours au RSA parmi les moins de 25 ans. De plus, alors que les jeunes non-insérés de ce groupe d'âges étaient principalement des hommes, le recours au RSA avant 25 ans est majoritairement féminin.

Rappelons qu'avant 25 ans, le RSA «classique » n'est ouvert qu'aux jeunes parents isolés assumant la charge d'au moins un enfant né ou à naître. Cette réglementation expliquerait la surreprésentation des femmes de moins de 25 ans au sein du RSA. Ces femmes composent des foyers monoparentaux avec peu ou pas de ressources, relativement "préservées » de l'exclusion par le régime du RSA. Dans tous les autres cas, les conditions d'attribution d'un RSA « jeune actif » doivent être remplies. En plus des critères généraux, le bénéficiaire devra alors justifier de deux ans d'activité en 
équivalent temps plein durant les trois années précédant la demande. À la fin de l'année 2015, le RSA jeune actif concernait moins de 7500 individus en France métropolitaine (données CafData).

Figure 5. Évolution 2011-2015 des « taux » de bénéficiaires du RSA par sexe et par groupe d'âges en Gironde

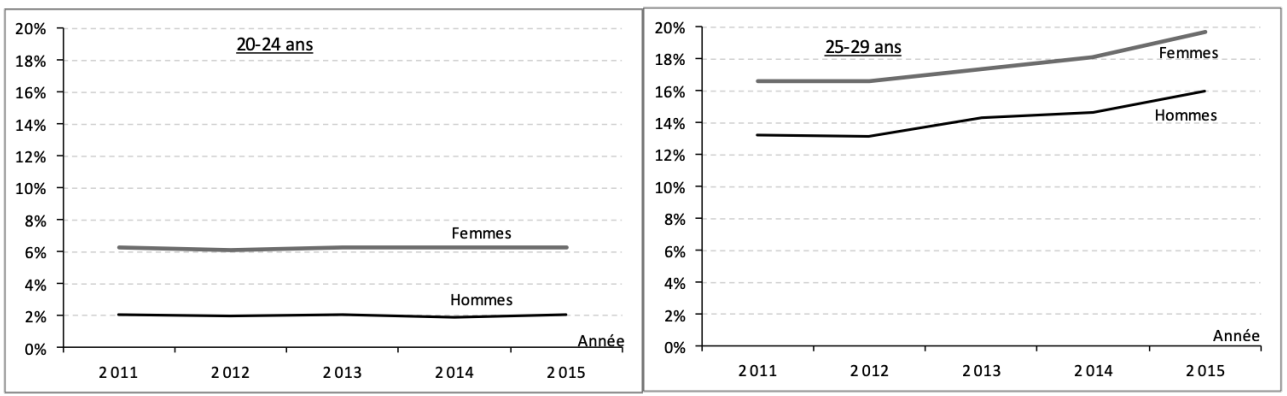

Traitement : auteurs

Source : Conseil départemental de la Gironde, extraction SID/INSEE-Enquêtes annuelles de recensement 2011 à 2015

19 En Gironde, seuls 203 jeunes percevaient le RSA jeune en 2015. Concernant moins de $0,2 \%$ des 18-24 ans depuis sa mise en place en 2010, aussi bien en France métropolitaine qu'en Gironde, le RSA jeune reste donc un dispositif très peu sollicité. Différentes hypothèses peuvent expliquer le faible recours, comme la propension des bénéficiaires potentiels du RSA jeune à être proches de l'emploi et donc à entrer et sortir rapidement du dispositif ou la forte proportion de jeunes encore scolarisés à ces âges-là. De toutes les explications, la plus plausible semble être la rigueur de la condition d'activité pour l'attribution de ce RSA jeune actif. Cette condition restreint le dispositif aux jeunes entrés rapidement sur le marché du travail et aujourd'hui sans emploi et sans droits au chômage. Compte tenu de ces obstacles, la situation des jeunes hommes de moins de 25 ans pose question. Il apparaît ici qu'ils ne bénéficient pas ou très peu du RSA (classique ou jeune actif) avant 25 ans, et par ailleurs, comme constaté précédemment, ils sont plus souvent non insérés que les femmes du même âge.

\section{Quel renouvellement de la population de bénéficiaires du RSA en Gironde?}

20 L'approche transversale (étude des évolutions année par année) nous a montré que depuis sa mise en place, le «stock» de jeunes bénéficiant du RSA est en augmentation. D'un point de vue " comptable ", ce stock mesuré à l'issue de chaque année dépend à la fois du stock de l'année précédente et des flux d'entrées et de sorties enregistrés au cours de l'année. À partir de ces éléments il est possible de calculer des taux d'entrée et de sortie de la population étudiée.

21 L'analyse des taux d'entrée et de sortie d'une population permet de mesurer sa dynamique. Les taux de sortie traduisent ainsi le fait de quitter, de manière plus ou moins définitive, une situation pour en intégrer une autre. Dans le cas du RSA, la "volatilité » des sorties et des entrées appelle une grande prudence en matière d'interprétation des rythmes de renouvèlement de la population des bénéficiaires du RSA. Un bénéficiaire peut ainsi effectuer plusieurs sorties et plusieurs entrées au cours 
de la même année. Une partie de ces sorties sont d'ordre administratif (radiation suite au non-respect d'une obligation et réintégration par la suite) et ne reflètent pas un changement réel et durable de situation.

Pour remédier à cette volatilité et mieux appréhender le renouvellement de cette population, nous avons alimenté l'approche transversale par une approche longitudinale. Par un suivi de cohorte, nous avons ainsi calculé les proportions de bénéficiaires présents dans le dispositif selon la durée écoulée depuis leur entrée. Ces proportions sont présentées pour différentes cohortes d'entrée et selon l'âge à l'entrée dans la série de graphiques ci-dessous.

Figure 6. Proportions des entrants présents dans le dispositif, selon la durée écoulée et la cohorte d'entrée au RSA en Gironde
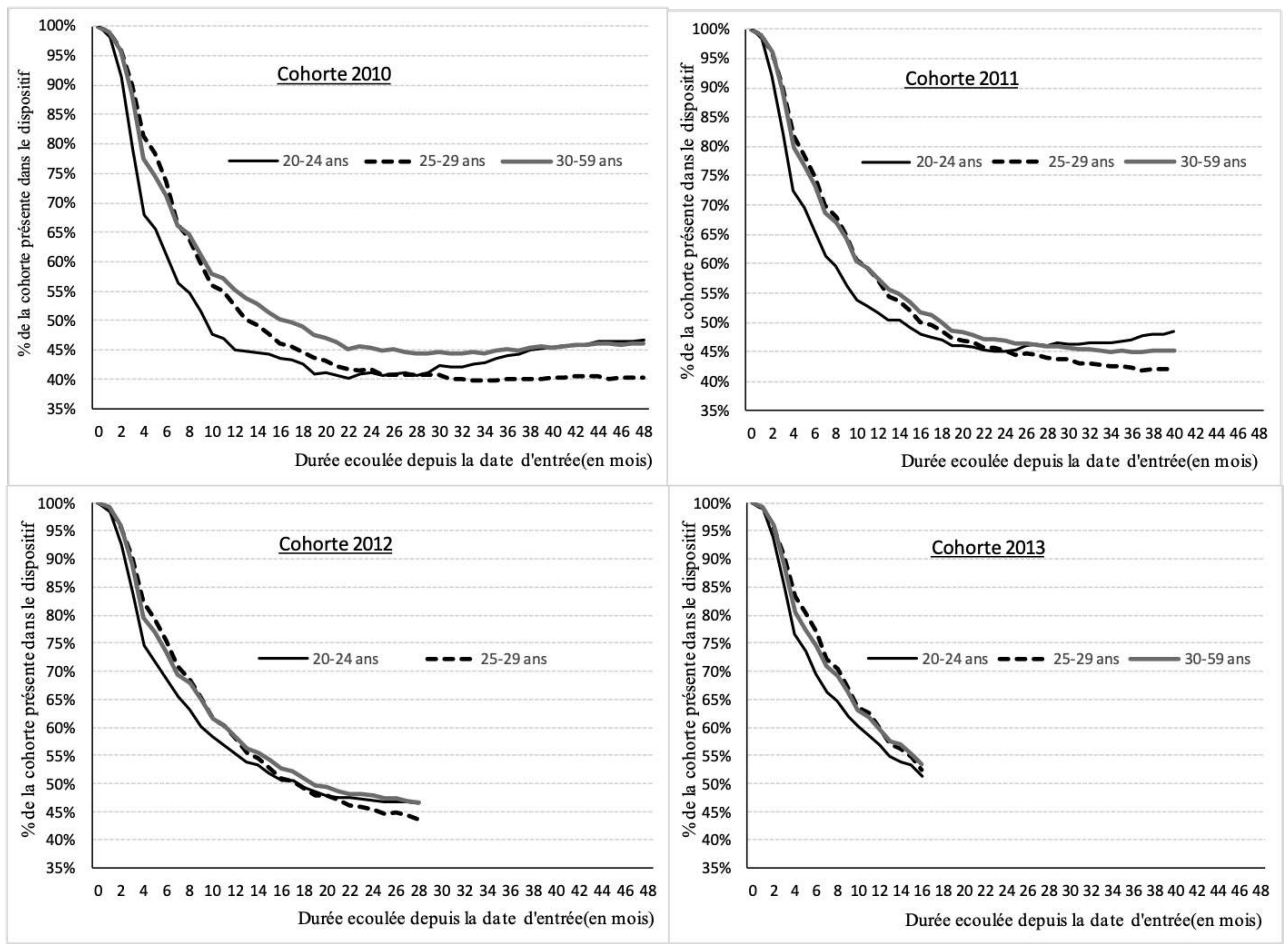

Traitement : auteurs

Source : Conseil départemental de la Gironde, extraction SID 2010 à 2013

Plusieurs éléments ressortent des suivis de cohortes de bénéficiaires du RSA en Gironde. On constate tout d'abord que, pour une cohorte et un groupe d'âges donnés, la proportion des présents ne baisse quasiment plus au-delà de vingt-quatre mois de présence dans le dispositif. Par ailleurs, le rythme de sortie du dispositif durant les deux années suivant l'entrée varie sensiblement selon l'âge au moment d'intégrer le RSA. Sur ce point, il apparaît que :

- la sortie se fait plus rapidement pour les plus jeunes. Cependant, pour les 20-25 ans on observe une réintégration du RSA après l'avoir quitté, au-delà de vingt-quatre mois après la date de la première entrée ;

- parmi l'ensemble des entrants, ce sont les jeunes de 25-29 ans qui sortent le plus et de manière définitive du dispositif RSA. Ainsi, douze mois après y être entrés, près de $50 \%$ des entrants de ce groupe ont quitté le RSA. Cette proportion atteint son niveau définitif de $60 \%$ vingt-quatre mois après la date d'entrée ; 
- parmi les 30-59 ans, les sorties se font plus lentement. Ainsi il faut attendre dix-huit mois pour voir un bénéficiaire sur deux (50\%) quitter le RSA après y être entré ;

- enfin, quel que soit le groupe d'âges, plus la cohorte d'entrée est récente et plus le rythme de sortie du RSA est ralenti. Ce constat s'accorde avec les résultats précédents à savoir une augmentation constante du «stock » de bénéficiaires depuis 2009. Il apparaît en outre que le ralentissement du rythme de sortie entre la première cohorte de 2010 et la dernière de 2013 est d'autant plus marqué que l'âge à l'entrée est bas.

\section{Les jeunes et le RSA : transition conjoncturelle?}

d'identifier quels sont les facteurs pouvant expliquer les évolutions observées auparavant. Parmi ces facteurs, l'élément déclencheur de la demande du RSA joue un rôle déterminant dans la «destinée » du demandeur. On peut distinguer les facteurs ayant une dimension conjoncturelle (temporaire) de ceux ayant une dimension structurelle (plus ou moins durable). Dans le premier groupe on trouve par exemple la situation de transition entre la fin des études et l'intégration effective sur le marché de l'emploi. Dans le second groupe de facteurs (structurels), on peut prendre pour exemple les changements touchant la situation familiale (arrivée d'un enfant, divorce, etc.).

Figure 7. Répartition selon le groupe d'âges des situations au moment de l'entrée au RSA en Gironde en 2015

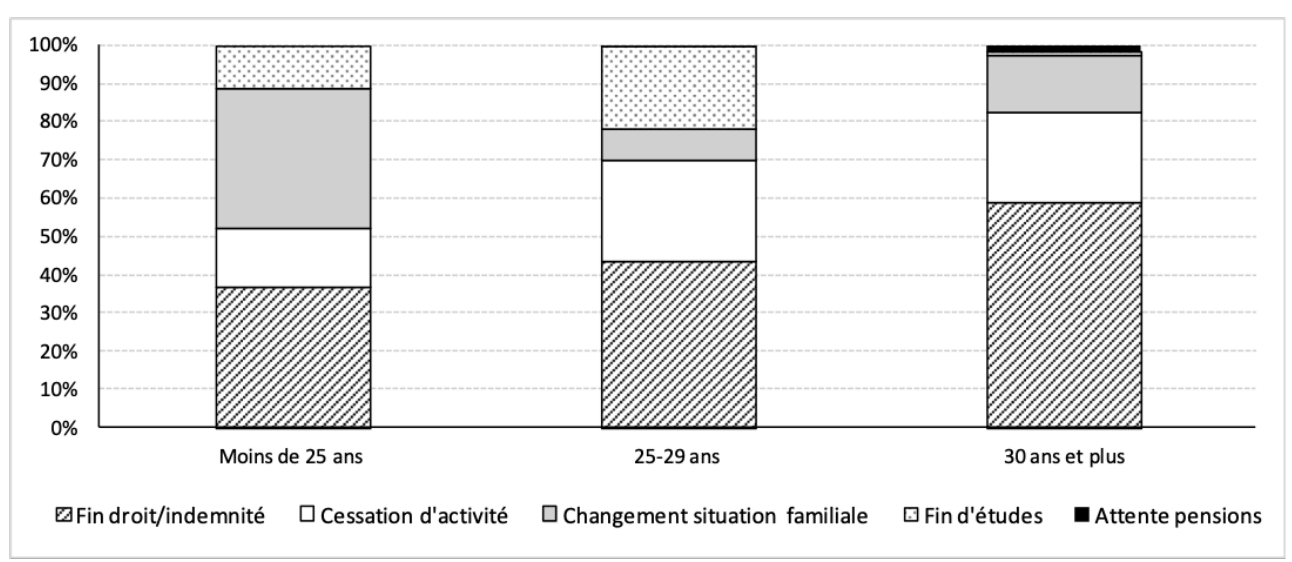

Traitement : auteurs

Source : Conseil départemental de la Gironde, extraction SID 2015

De la lecture de ce graphique il ressort que les facteurs déclenchant la demande de RSA chez les jeunes de 25-29 ans semblent davantage d'ordre conjoncturel (fin de droits, cessation d'activité, transition entre études et emploi). Chez ces jeunes qui, rappelonsle, sont ceux qui quittent le plus et de manière définitive le RSA, le changement de la 
situation familiale est relativement moins présent (8\%) comparativement à ce qu'on observe chez les autres groupes d'âges.

En lien avec ces différences de situation au moment de demander le RSA, les niveaux de diplôme les plus élevés sont enregistrés chez les jeunes qui ont entre 25 et 29 ans lors de la demande du RSA. Dans ce groupe d'âge, six personnes sur dix ont au moins le bac, proportion qui se situe entre $25 \%$ et $45 \%$ dans les autres groupes d'âges (Figure 8).

Figure 8. Répartition des entrants au RSA en 2015 selon le niveau de diplôme et l'âge au moment de l'entrée au RSA

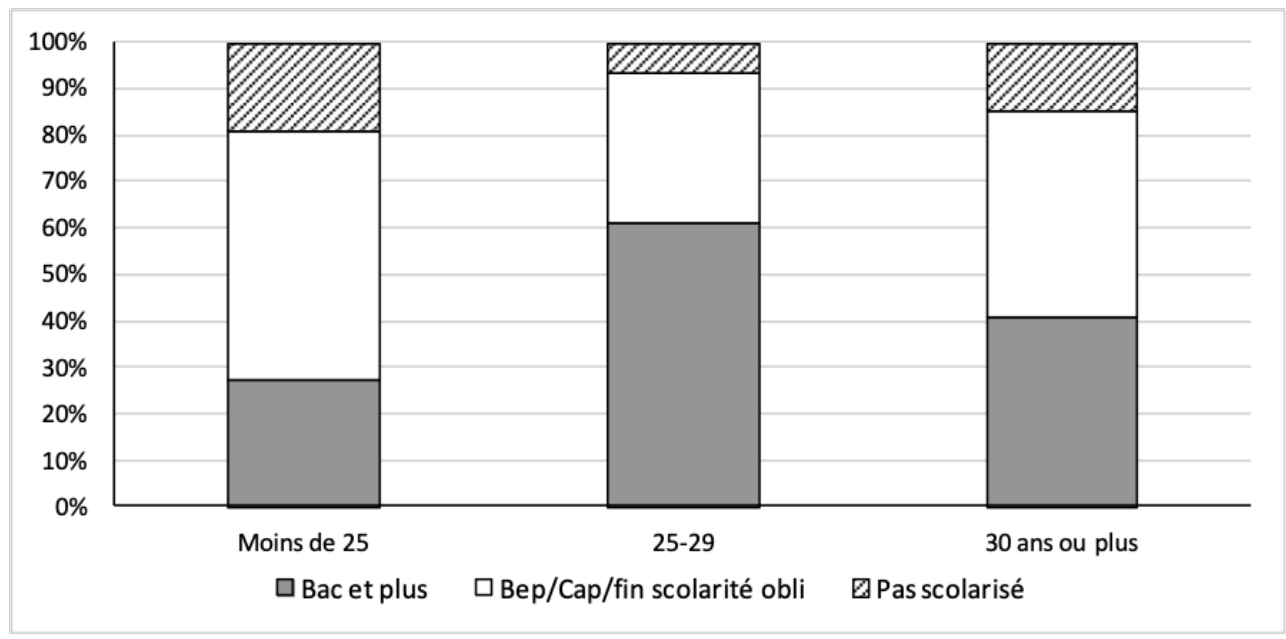

Traitement : auteurs

Source : Conseil départemental de la Gironde, extraction SID 2015

Cette logique de situation transitionnelle (fin d'études ou cessation d'activité) chez les jeunes inscrits au RSA est confirmée par la «fraicheur » de leur inscription sur les listes de demandeurs d'emploi. En effet, plus d'un demandeur de 25 à 29 ans sur deux est à la recherche d'un emploi depuis moins d'un an. En outre, $71 \%$ des jeunes de ce groupe d'âge déclarent n'avoir aucun obstacle pour chercher un emploi, alors que moins de six personnes sur dix sont dans ce cas de figure dans les autres classes d'âges.

$\mathrm{Au}$ total, ces analyses montrent que les facteurs qui poussent les jeunes de 25-29 ans à intégrer le RSA sont davantage conjoncturels que structurels. Il s'agit également du groupe d'âge au sein duquel les conditions susceptibles de faire sortir les personnes du RSA sont davantage réunies. Ces éléments sont déterminants dans la réponse à apporter aux jeunes de moins de 30 ans qui connaissent un ou plusieurs épisodes de RSA, dans la mesure où l'action sur des facteurs conjoncturels est généralement plus rapide, surtout quand les conditions qui permettent d'écourter la période de la transition sont réunies.

\section{Conclusion}

La dernière crise, si elle explique une partie du contexte, ne peut résumer les récentes mutations professionnelles et sociales auxquelles font face les jeunes adultes en France. Comme les enquêtes Génération menées par le Centre d'études et de recherche sur les qualifications (CEREQ) le rappellent régulièrement, le diplôme continue d'être un facteur d'employabilité fort. Mais en dépit de l'élévation du niveau de diplôme au cours 
des dernières décennies, l'exclusion et la précarisation touchent un nombre croissant de jeunes, diplômés ou non, et aux origines sociales variées (DARES et France Stratégie, 2017).

Le lien traditionnellement étroit entre emploi et insertion, réaffirmé dans les ambitions du dispositif du RSA, est l'hypothèse de travail qui a animé cette mise en perspective de la non-insertion et du recours au RSA. Les jeunes adultes de moins de 30 ans constituent un public adapté à l'analyse, tant ils sont à la fois surexposés au risque d'exclusion et grandement présents dans le dispositif RSA.

Comme l'a montré l'exemple girondin, la non-insertion scolaire et professionnelle des jeunes adultes a pris de l'ampleur ces dernières années. Alors que les femmes de moins de 25 ans semblent mieux préservées de l'exclusion que les hommes, la tendance se renverse chez les 25-29 ans. Indépendamment du sexe, les jeunes de plus de 25 ans sont cependant plus souvent en situation d'exclusion que les plus jeunes. Ces constats appellent de nombreuses questions sur les processus qui mènent à cette non-insertion et notamment la rupture prématurée avec l'école ainsi que les perspectives d'emploi offertes aux jeunes de ces âges.

La dégradation des conditions de travail, notamment parmi les moins diplômés (Aliaga et Lê, 2016), est une composante essentielle de l'emploi précaire chez les jeunes. La réponse apportée par le RSA jeune semble ne pas correspondre aux difficultés de la majorité des jeunes en situation de précarité. Ce sont notamment la durée et la stabilité de la transition des jeunes de l'école au monde du travail qui posent question. Par l'observation de cohortes de jeunes adultes qui ont été ou sont toujours au RSA, il apparaît qu'une partie croissante de ce public retourne lentement et difficilement à un emploi stable. La contextualisation laisse cependant penser que le public des jeunes, et en particulier des 25-29 ans, est un public relativement proche de l'emploi et faisant principalement face à des blocages conjoncturels. La résolution de ces différentes problématiques constitue un enjeu d'importance, d'abord car la précarité peut constituer une étape aux conséquences durables sur le parcours de vie, d'autant plus lorsqu'elle survient aux jeunes âges de la vie active (Lima et Touriah-Gaillard, 2012). De plus, en termes de dynamiques locales, les jeunes adultes qui ne s'insèrent pas rapidement et de manière stable s'ajoutent progressivement aux nouveaux diplômés sur le marché de l'emploi des débutants, y accentuant la tension.

Observées au niveau girondin, les tendances de retour à l'emploi des jeunes adultes ayant connu le RSA sont d'autant plus parlantes qu'elles sont observées à plus grande échelle. Le système de protection sociale actuel s'articule autour d'une catégorisation par l'âge. Or il apparaît aujourd'hui que le principal élément de distinction entre un "jeune» et un "adulte» est la stabilité de la situation professionnelle, ainsi que l'émergence dans le parcours de vie de l'individu des processus d'autonomisation qui y sont liés. Bargain et Vicard ayant établi que l'octroi du RSA aux moins de 30 ans n'était pas source de désincitation au travail (Bargain et Vicard, 2014) et compte tenu des bonnes prédispositions des jeunes de ces âges à s'insérer sur le marché de l'emploi, il apparaît que l'État social doit continuer de soutenir les moins de 30 ans dans leur parcours de stabilisation. À ce titre, le Compte personnel d'activité, ou encore la Garantie jeunes, sont des dispositifs de structuration des parcours qui préfigurent la protection sociale des jeunes d'aujourd'hui et de demain. 


\section{BIBLIOGRAPHIE}

Aliaga C. et LÊ J. (2016), « L'insertion des jeunes sur le marché du travail : l'emploi est majoritaire chez les plus diplômés, l'inactivité domine chez les non-diplômés », in France, portrait social, p. 43-58.

Allegre G. (2011), « La jeunesse, génération sacrifiée? », Les notes du blog, n 5, p. 1-12, disponible sur : http://www.ofce.sciences-po.fr/pdf/notes/2011/note5.pdf.

Arrighi J.-J. (2012), Quand l'école est finie... Premiers pas dans la vie active d'une génération. Enquête 2010, Marseille, CEREQ, 63 p.

Bargain O. et Vicard A. (2014), « Le RMI et son successeur le RSA découragent-ils certains jeunes de travailler? Une analyse sur les jeunes autour de 25 ans ", Économie et statistique, n 467-468, p. 61-89.

Bergouignan C. et Rebière N. (2013), " Définir les travailleurs pauvres pour les dénombrer ? ", in Auvergnon P. (dir.), Droit social et travailleurs pauvres, Louvain-la-Neuve, Bruylant, p. 69-95.

Bonnet C. (2010), « Niveau de vie : un rattrapage des jeunes générations ? », Regards croisés sur l'économie, vol. 7, n 1, p. 50-55.

Comité national d'évaluation du RSA (2011), Rapport final, 150 p.

Cordazzo P. et Sembel N. (2012), « Du Master à l'aide sociale : déclassement immédiat, “piège statutaire", nouvelles trajectoires de précarisation ", L'orientation scolaire et professionnelle, vol. 41, $\mathrm{n}^{\circ} 1$, disponible sur : http://osp.revues.org/3703.

DARES et France Stratégie (2017), «L'insertion professionnelle des jeune », rapport de diagnostic, $68 \mathrm{p}$.

Dubar C. (1987), L'autre jeunesse. Des jeunes sans diplôme dans un dispositif de socialisation, Lille, Presses universitaires de Lille, $263 \mathrm{p}$.

Lima L. (2012), « Les jeunes vulnérables : laboratoire de l'État social actif », in Labadie F. (dir.), Inégalités entre jeunes sur fond de crise, Rapport de l'observatoire de la jeunesse 2012, Paris, La Documentation Française, p. 184-198.

Lima L. et Touriah-Gaillard A. (2012), « Vulnérables et vulnérabilités », in Lima L. et Trombert C. (dir.), Le travail de conseiller en insertion, Montrouge, ESF éditeur.

Magord C. (2017), « Du RMI jeunes à la Garantie jeunes : les débats sur le soutien à l'autonomie des jeunes adultes », Informations sociales, $n^{\circ} 195$, p. 66-75.

Minni C. (2015), « Emploi et chômage des 15-29 ans en 2014 : stabilisation des taux d'activité, d'emploi et de chômage ", DARES Analyses, $\mathrm{n}^{\circ}$ 88, $12 \mathrm{p}$.

Observatoire girondin de la précarité et de la pauvreté (2016), Rapport 2016, 132 p.

OCDE (2016), « The NEET challenge: What can be done for jobless and disengaged youth? », in Society at a Glance 2016, Paris, OECD Publishing, p. 13-68. 


\section{NOTES}

1. Les auteurs remercient le Conseil départemental de la Gironde d'avoir permis l'accès à des données sans lesquelles cette recherche n'aurait pas été possible.

2. Les bénéficiaires regroupent à la fois les allocataires et leurs conjoints.

3. Nous ne disposons pas de données consolidées de la répartition par âges des bénéficiaires du RSA en 2009 et 2010.

\section{RÉSUMÉS}

Entre études supérieures, exclusion, intérim, chômage et réorientation, l'insertion professionnelle des jeunes est un processus de moins en moins linéaire et prévisible. Les récents débats autour de la « désincitation au travail » ont entraîné la refonte d'une partie du système de protection sociale en conditionnant l'aide publique à l'emploi, et ce de manière encore plus stricte lorsqu'il s'agit de l'octroyer à un(e) jeune de moins de 30 ans. Cette mutation de l'état social s'appuie sur une nouvelle perception de la solidarité dite " active ", dont un des dispositifs les plus symboliques est le Revenu de solidarité active (RSA). En s'intéressant à la fois aux jeunes non-insérés et à ceux au RSA, dont les trajectoires relèvent de processus complexes, cette étude mêle données de contextualisation et suivi de cohorte afin de déterminer les récentes tendances de l'insertion des précaires de moins de 30 ans en Gironde ${ }^{1}$.

\section{INDEX}

Mots-clés : précarité, insertion professionnelle, RSA

\section{AUTEURS}

\section{MEHDI LE PETIT-GUERIN}

Doctorant en démographie

Université de Bordeaux, COMPTRASEC-UMR 5114

\section{KHALID ELJIM}

Chargé d'analyse et de prospective au département de la Gironde Chercheur associé au COMPTRASEC-UMR 5114 